\title{
Tertiary Gleason Pattern
}

National Cancer Institute

\section{Source}

National Cancer Institute. Tertiary Gleason Pattern. NCI Thesaurus. Code C48605.

Prostate carcinoma patterns occupying less than $5 \%$ of the tumor. 